\title{
Plant Mitochondrial DNA Evolves Rapidly in Structure, but Slowly in Sequence
}

\author{
Jeffrey D. Palmer and Laura A. Herbon \\ Department of Biology, University of Michigan, Ann Arbor, Michigan 48109, USA
}

\begin{abstract}
Summary. We examined the tempo and mode of mitochondrial DNA (mtDNA) evolution in six species of crucifers from two genera, Brassica and Raphanus. The six mtDNAs have undergone numerous internal rearrangements and therefore differ dramatically with respect to the sizes of their subgenomic circular chromosomes. Between 3 and 14 inversions must be postulated to account for the structural differences found between any two species. In contrast, these mtDNAs are extremely similar in primary sequence, differing at only 1-8 out of every $1000 \mathrm{bp}$. The point mutation rate in these plant mtDNAs is roughly 4 times slower than in land plant chloroplast DNA (cpDNA) and 100 times slower than in animal mtDNA. Conversely, the rate of rearrangements is extraordinarily faster in plant mtDNA than in cpDNA and animal mtDNA.
\end{abstract}

Key words: Genome evolution - Rearrangement - Inversion - Brassica - Base substitution

\section{Introduction}

Mitochondrial and chloroplast genomes contain two vital sets of genes. First, they encode many proteins crucial to the fundamental bioenergetic processes of the cell. Second, they encode many of the components necessary for the proper expression of their own genes. Given the vital importance of these two sets of organellar genes, one might expect them to change very slowly during the course of evolution. Indeed, chloroplast genes have a low rate of point mutations (reviewed in Palmer 1985a; Wolfe et al.

Offprint requests to: J.D. Palmer
1987; Zurawski and Clegg 1987). Animal mitochondrial DNA (mtDNA), however, has a very rapid rate of point mutations. First clearly measured for primates (Brown et al. 1979), this rapid rate now appears to be a general feature of most groups of vertebrates (reviewed in Brown 1983, 1985; Wilson et al. 1985).

Sequence studies of several plant mitochondrial genes suggest a much lower rate of point mutations than in animal genomes (reviewed in Palmer 1985a; Sederoff 1987; Wolfe et al. 1987). However, several factors limit the conclusions that could be derived from such studies: (1) Comparison of a few kilobases of gene sequences provides little insight upon mutation rates for the entire genome, which in plants is quite large (Ward et al. 1981) and mostly noncoding (Makaroff and Palmer 1987). (2) These sequence comparisons have mostly been between distantly related angiosperms; comparisons between more closely related species are likely to yield more precise and informative measurements of evolutionary patterns and rates (Brown et al. 1982). (3) None of these studies have allowed adequate comparisons with rates of change in chloroplast and nuclear genomes from the same groups of closely related plants.

Rates of sequence and structural evolution can be remarkably different for a given organelle genome. Animal mtDNAs, which change so rapidly in primary sequence, are essentially invariant in gene order among all vertebrates (Brown 1983, 1985). Land plant chloroplast DNAs (cpDNAs) evolve slowly in base sequence and rarely undergo internal rearrangement (Palmer 1985a,b). Plant mtDNAs may also change slowly in sequence, however, they are extremely variable in size $(200-2400 \mathrm{~kb}$; Ward et al. 1981; Palmer 1985a; Pring and Lonsdale 1985), 
contain numerous pieces of cpDNA (Lonsdale 1988), and usually exist as a collection of different-sized circles (Lonsdale 1988). This diversity implies a high rate of length mutations (deletions, insertions, duplications). However, there is little information available concerning rates of inversions and transpositions, particularly as measured over the entire plant mitochondrial genome. Extensive internal rearrangement has been suggested for mtDNAs in Zea (Sederoff et al. 1981) and Nicotiana (Bland et al. 1985), but the evidence is limited to inferences from Southern blot hybridizations, in the absence of any supporting physical maps. Specific mtDNA rearrangements have been characterized in great detail in several plants (Schardl et al. 1985; BaileySerres et al. 1986; Dewey et al. 1986; Young and Hanson 1987), but these events have not been placed in the larger context of genome-wide rates and processes of rearrangement.

Here, we provide the first measurements of the tempo and mode of evolutionary change over the entire mitochondrial genome in a group of closely related plants. We have chosen to study species in the genus Brassica ${ }^{1}$ for several reasons. First, the relatively small size (208-242 kb) of the mitochondrial genome in Brassica considerably facilitates analysis of the entire genome (Palmer and Shields 1984). Second, compared to other groups of plants (Ward et al. 1981), genome size is relatively invariant in Brassica (Lebacq and Vedel 1981; Palmer et al. 1983b), making it easier to study processes of rearrangement and point mutation in the absence of the confounding effects of extensive length mutation. Third, the tempo and mode of cpDNA evolution are already worked out for Brassica species (Palmer et al. 1983a), enabling direct comparisons of evolutionary processes in the two plant cytoplasmic genomes.

We report a remarkable and novel pattern of evolutionary change in Brassica mitochondrial genomes. Compared to all previously studied organelle genomes, these molecules change extraordinarily slowly in base sequence, yet very rapidly in structure and arrangement.

\section{Materials and Methods}

Mitochondrial DNA was isolated from 6-wk-old green leaves of Brassica campestris cv. Purple Top White Globe (turnip), $B$. napus cv. American Purple Top (rutabaga), B. oleracea cv. Brunswick (cabbage), $B$. hirta (white mustard; USDA PI 195, 922), $B$. nigra (black mustard; 84/row 1307 , gift of $D$. Cohen), and $R a-$

\footnotetext{
"We shall use "Brassica" to refer to all five species compared herein that are classified in the genus Brassica and also for Raphanus sativus (radish), because cytoplasmically it too belongs in Brassica (Palmer et al. 1983a)
}

phanus sativa cv. Scarlet Knight (fertile radish) by the DNase I procedure of Kolodner and Tewari (1972). Restriction endonuclease digestions, agarose gel electrophoresis, bidirectional blot transfers to Genescreen (New England Nuclear) and Zetabind (AMF Cuono) hybridization membranes, nick-translations, filter hybridizations, and cloning of mtDNA fragments into plasmid vectors were performed as described (Palmer and Shields 1984; Palmer 1986). Complete physical maps were constructed for mtDNAs of $B$. oleracea and $B$. napus according to a previously described mapping strategy (Palmer and Shields 1984). Probes used in mapping hybridizations consisted of plasmids containing almost the entire $B$. campestris mitochondrial genome (Palmer and Shields 1984) and selected portions of the $B$. oleracea genome, and also gel-isolated fragments from both the $B$. oleracea and $B$. napus genomes.

\section{Results}

\section{Tricircular Mitochondrial DNAs}

We previously showed that the mitochondrial genomes of three of the six species examined here, $B$. campestris, $B$. nigra, and Raphanus sativa, exist in a tripartite organization as the result of intragenomic recombination across a pair of direct repeats. This recombination interconverts a master chromosome with two subgenomic circles (Palmer and Shields 1984; Palmer and Herbon 1986). In contrast, $B$. hirta mtDNA lacks any recombination repeats ${ }^{2}$ and so exists as a single circular chromosome (Palmer and Herbon 1987). Furthermore, different sequences are repeated and recombine in $B$. campestris relative to $B$. nigra and $R$. sativa (Palmer and Herbon 1986). We have now investigated the recombinational activity of mtDNAs from $B$. oleracea and $B$. napus and constructed complete restriction maps of their genomes (Fig. 1) to allow evolutionary comparisons among all six of these Brassica mtDNAs.

These mapping studies reveal that the same $2-\mathrm{kb}$ element that is duplicated as a direct repeat and engaged in intramolecular recombination in $B$. campestris mtDNA (Palmer and Shields 1984) has a similar organization in both $B$. oleracea and $B$. napus (Fig. 1). This implies a similar tricircular structure for all three genomes (Fig. 2). Note, however, two major differences in the organization of the recombination repeats in the three mtDNAs. First, although overall genome size is highly conserved, at $218 \mathrm{~kb}$ for $B$. campestris, $219 \mathrm{~kb}$ for $B$. oleracea,

\footnotetext{
${ }^{2}$ We use the term "recombination repeat" as defined previously (Stern and Palmer 1984) to denote a sequence present in two copies relative to unique sequences in the genome, but which exists in four different genomic environments as the result of recombination occurring at high frequency (sufficient to produce an equilibrium mixture consisting of essentially equal levels of the recombinational isomers) between the two repeat copies. This operational definition does not include short repeats and other sequences that only recombine rarely
} 


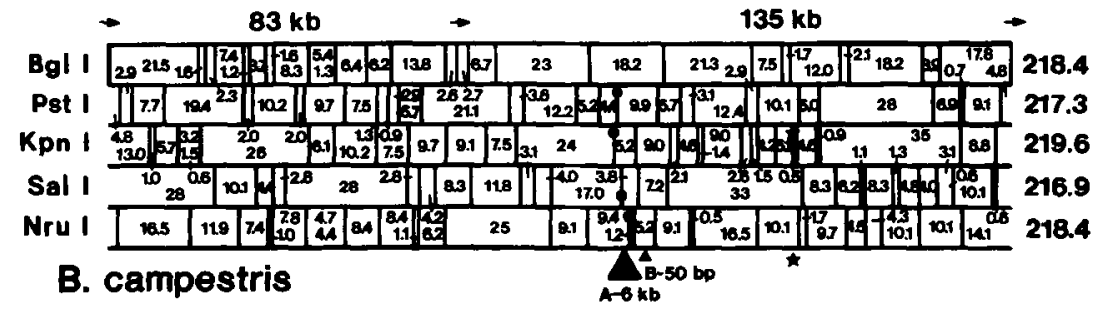

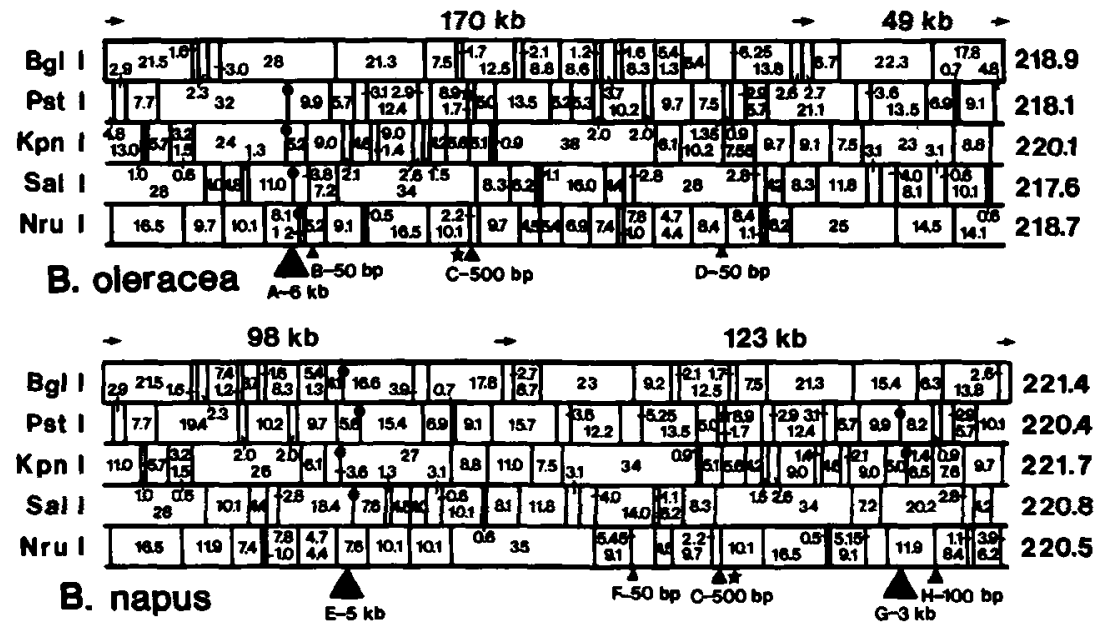

Fig. 1. Restriction maps of the master chromosomes of three Brassica mtDNAs. The circular maps are shown linearized at a Bgll site within the 2-kb repeat. Arrows indicate the two copies of this repeat.

Numbers between arrows indicate sizes of subgenomic circles (cf. Fig. 2). Restriction fragment summations are given at the right of the maps. Filled triangles indicate eight length mutations, which are arbitrarily shown only as insertions. Variable restriction sites resulting from length mutation and point mutation are indicated with filled circles and stars, respectively. The $B$. campestris map is modified from Palmer and Shields (1984).

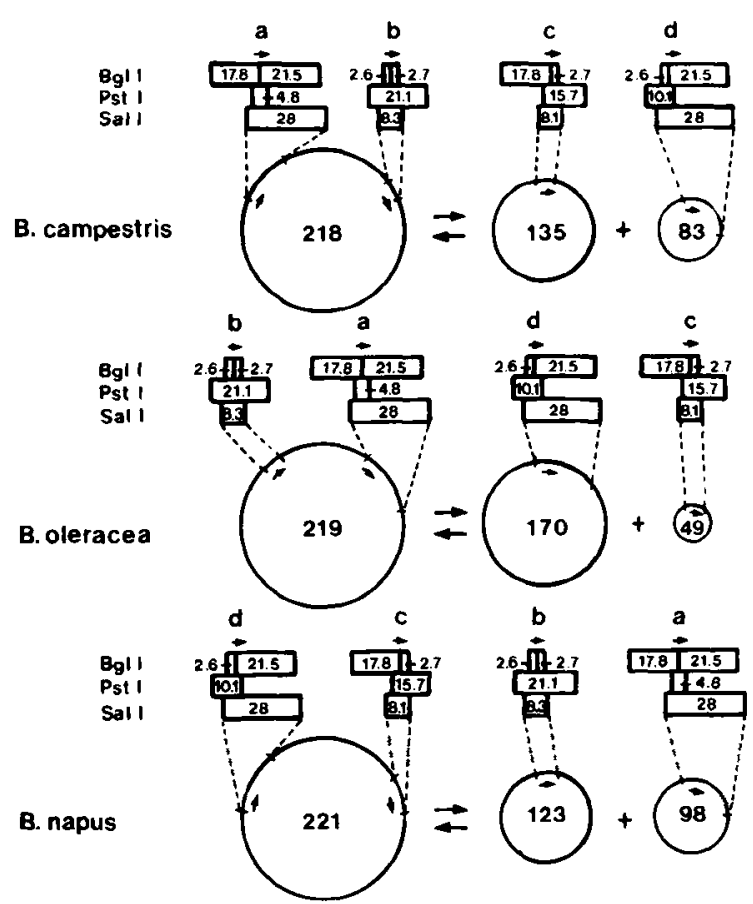

Fig. 2. Tricircular organization of three Brassica mtDNAs. Arrows denote the position and orientation of a $2-\mathbf{k b}$ sequence that is duplicated and engaged in high frequency intragenomic recombination in each mtDNA. Letters distinguish each repeat in terms of its configuration of flanking unique sequences. Cleavage maps for three restriction enzymes are shown in regions encompassing each of the repeats.

and $221 \mathrm{~kb}$ for $B$. napus, distances between repeats are different. This results in subgenomic circles of unequal size in $B$. oleracea (170 kb and $49 \mathrm{~kb}$ ), whereas those in $B$. campestris (135 kb and $83 \mathrm{~kb}$ ) and $B$. napus (123 kb and $98 \mathrm{~kb})$ are more similar (Fig. 2). Second, different repeat configurations are present on different circles in one species relative to another. (There are two copies of the repeat per genome equivalent, but recombination places these in a total of four configurations with respect to unique flanking sequences.) The " $a$ " and " $b$ " configurations are present on the master chromosome and the " $c$ " and " $d$ " configurations on the subgenomic circles in both $B$. campestris and $B$. oleracea, whereas in $B$. napus the reverse is true (Fig. 2). Moreover, in $B$. campestris the " $c$ " configuration is present on the larger subgenomic circle and the " $d$ " configuration on the smaller one, whereas the opposite holds for $B$. oleracea (Fig. 2). These differences in circle size and repeat location have the same cause, i.e., the inversions described below.

Our map for $B$. oleracea mtDNA agrees with that reported by Chetrit et al. (1984) in the placement of $90-95 \%$ of the sites for the three enzymes mapped in common. However, Chetrit et al. describe only a single master chromosome, on which they place our " $a$ " and " $c$ " configuration repeats, whereas according to our mapping studies these two repeat configurations can only exist on different genome isomers and never on the same chromosome. Because the $B$. oleracea mtDNAs show identical patterns with the three enzymes used in common in the two studies, strain differences cannot account for these discrepancies. Therefore, we feel their map is in error, and in fact describes a highly improbable genome arrangement. 

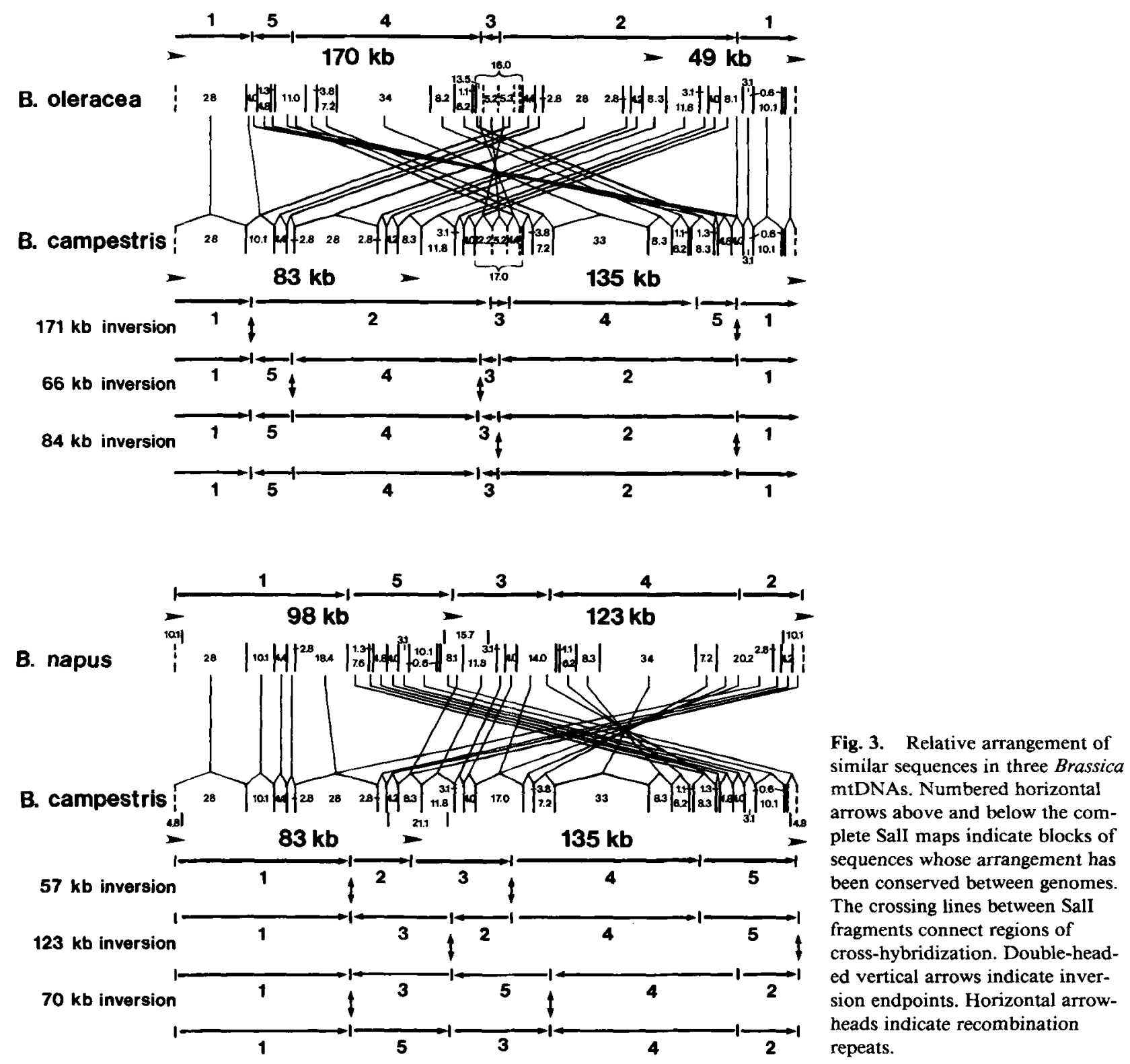

\section{Inversions in Brassica mtDNAs}

The relative arrangement of sequences in the six Brassica mtDNAs was assessed by hybridizing cloned fragments covering $96 \%$ of the $B$. campestris genome (Palmer and Shields 1984) to filter-bound mtDNAs from the other five species, each digested with the five enzymes used in mapping. In addition, selected clones from these five genomes were hybridized to filters containing digests of $B$. campestris mtDNA. We first compared the three most closely related mtDNAs, from $B$. campestris, $B$. oleracea, and $B$. napus. Alignment of cross-hybridizing SalI fragments from these three mtDNAs leads to two major conclusions (Fig. 3). First, the three genomes have essentially the same sequence content. Every fragment from the $B$. campestris reference genome hybridizes to fragments from the $B$. olerace $a$ and $B$. napus genomes, and vice versa. There are only a few detectable length mutations in these genomes, which will be discussed in a later section.

Second, the genomes differ significantly in linear sequence order. The $B$. campestris and $B$. oleracea genomes can be divided into five linkage groups, where sequences within each group have the same arrangement in the two genomes, but where the relative order and orientation of linkage groups differ between the two species (Fig. 3). A minimum of three separate inversions must be postulated to account for these linkage disruptions, i.e., to convert one genome arrangement into the other. The $B$. campestris $-B$. napus comparison also breaks the genomes into five linkage groups and postulates a minimum of three inversions (Fig. 3).

In each of the two comparisons, two of the postulated inversions share a common endpoint. For example, in the $B$. oleracea-B. campestris comparison, the right endpoints of the $171-\mathrm{kb}$ and $84-\mathrm{kb}$ 
inversions map to approximately the same position within a $B$. campestris probe fragment of $4.0 \mathrm{~kb}$. Only by sequencing these endpoints can we be certain of whether they fall precisely at the same place. Certain regions of the genome, perhaps containing short dispersed repeats, may promote inversional recombination.

Knowledge of the positions of inversions enables us to derive simple explanations for the differences noted in the preceding section regarding circle size and repeat location. The net effect of two successive inversions, each one flipping one repeat relative to the other (as diagrammed in each of the comparisons in Fig. 3), is to retain a direct orientation of the repeats on the master chromosome, but to move them either farther apart or closer together. Hence, a tricircular organization will be retained, but the sizes of the subgenomic circles will differ from one species to the other.

Such a two-step inversion of repeat-containing linkage groups postulates an evolutionary intermediate genome in which the repeats are oriented inversely relative to one another (Fig. 4). These genomes would be direct physical analogs of the "flipflop" inversion pairs found in cpDNA (reviewed in Palmer 1985a,b) and the 2-micron plasmid of yeast (Broach 1982). The two inversion isomers in the hypothesized intermediate genome are both "master chromosomes," i.e., they contain the entire sequence complexity of the mitochondrial genome. Therefore, the second evolutionary inversion in these two-step progressions is as likely to occur in the inversion isomer of the circle that sustained the first evolutionary inversion as in the unflipped circle. The net effect of this first outcome would be to generate a tricircular genome with direct repeats, in which the two repeat configurations of the original master chromosome are now on the subgenomes and vice versa. This is exactly the situation described in the preceding section for $B$. napus compared to the other two genomes (Fig. 2). Figure 4 shows an evolutionary model postulating exactly this series of events - a "flip-flop" inversion occurring in between (in a phylogenetic sense) two evolutionary inversions - the net effect of which is to convert the $B$. campestris genome into a $B$. napus genome.

The other three mtDNAs, from $B$. hirta, $B$. nigra, and $R$. sativa, are much more highly rearranged relative to each other and to the reference genome, from $B$. campestris, than are the three genomes compared thus far. Relative to $B$. campestris, these mtDNAs can be divided into no fewer than $11(B$. hirta; Palmer and Herbon 1987), 12 (B. nigra; Fig. 5 ), and 15 ( $R$. sativa; Fig. 5) blocks of linkage conservation. Rearrangement is so extensive in these genomes that we cannot derive a single unambiguous and parsimonious series of individual muta-

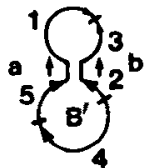

11
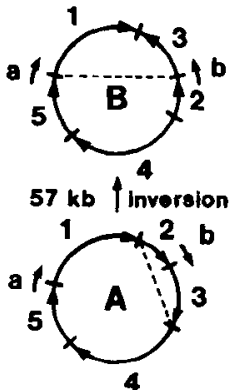

B. campestris

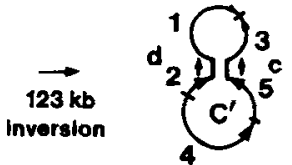

11

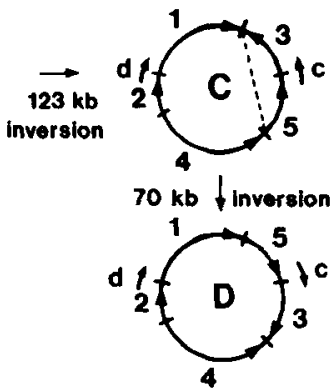

B. napus
Fig. 4. Evolutionary model for the interconversion of the $B$. campestris (A) and $B$. napus (D) mitochondrial genomes. Molecules $B$ and $C$ represent hypothesized evolutionary intermediates which themselves are interconvertible by a nonevolutionary inversion-a flip-flop inversion occurring within the paired recombination repeats as shown in the equivalent molecules $B^{\prime}$ and $C^{\prime}$. Numbers and arrows denote linkage groups shown in Fig. 3. Small letters denote the four configurations of the recombination repeat (cf. Fig. 2).

tions that would convert one genome form into another, as was feasible for the more closely related genomes (Figs. 3 and 4). If, by analogy to these simpler comparisons, we assume that all rearrangements (excluding length mutations) are inversions, then a minimum of 10,11 , and 14 inversions must be postulated to convert the $B$. campestris genome into a $B$. hirta, $B$. nigra, and $R$. sativa genome, respectively. If each of these putative inversions occurred independently (in location) of the others, then 20,22 , and 28 inversion endpoints would be predicted for each of the three comparisons. Because only 11,12 , and 15 rearrangement breakpoints were, in fact, detected (Fig. 5 and Palmer and Herbon 1987), many of the inversions must share common endpoints. This reinforces the conclusion derived above from the more straightforward and interpretable comparisons among the closely related Brassica species (Fig. 3) and suggests that certain regions of the mitochondrial genome are relatively active in promoting recombination.

\section{Nucleotide Sequence Divergence}

We estimated levels of nucleotide sequence divergence among the six mtDNAs based on the proportion of shared restriction sites. Comparisons over the entire genome were possible only for the three most closely related mtDNAs, from $B$. campestris, $B$. oleracea, and $B$. napus. Excluding 10 sites located within regions that are not present in all three genomes, a total of 133 mapped sites could be com- 


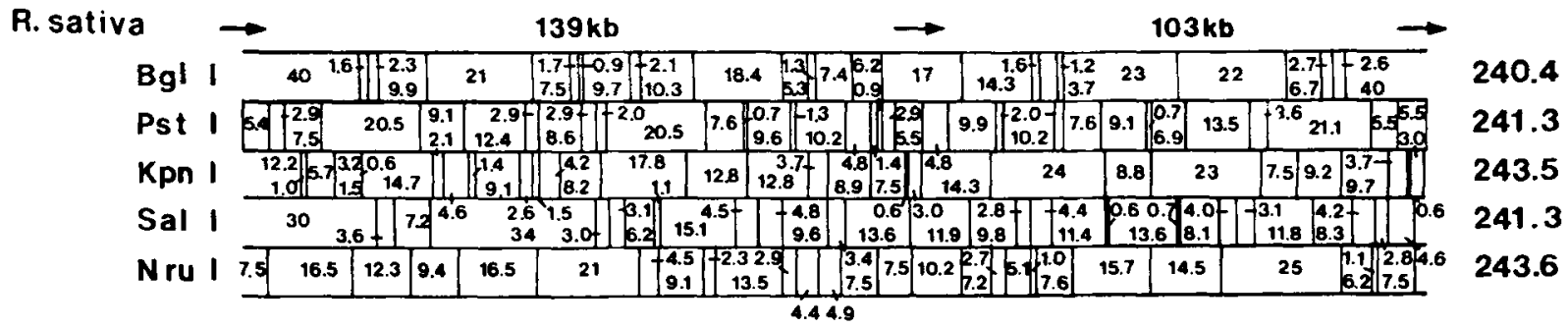

B. campestris
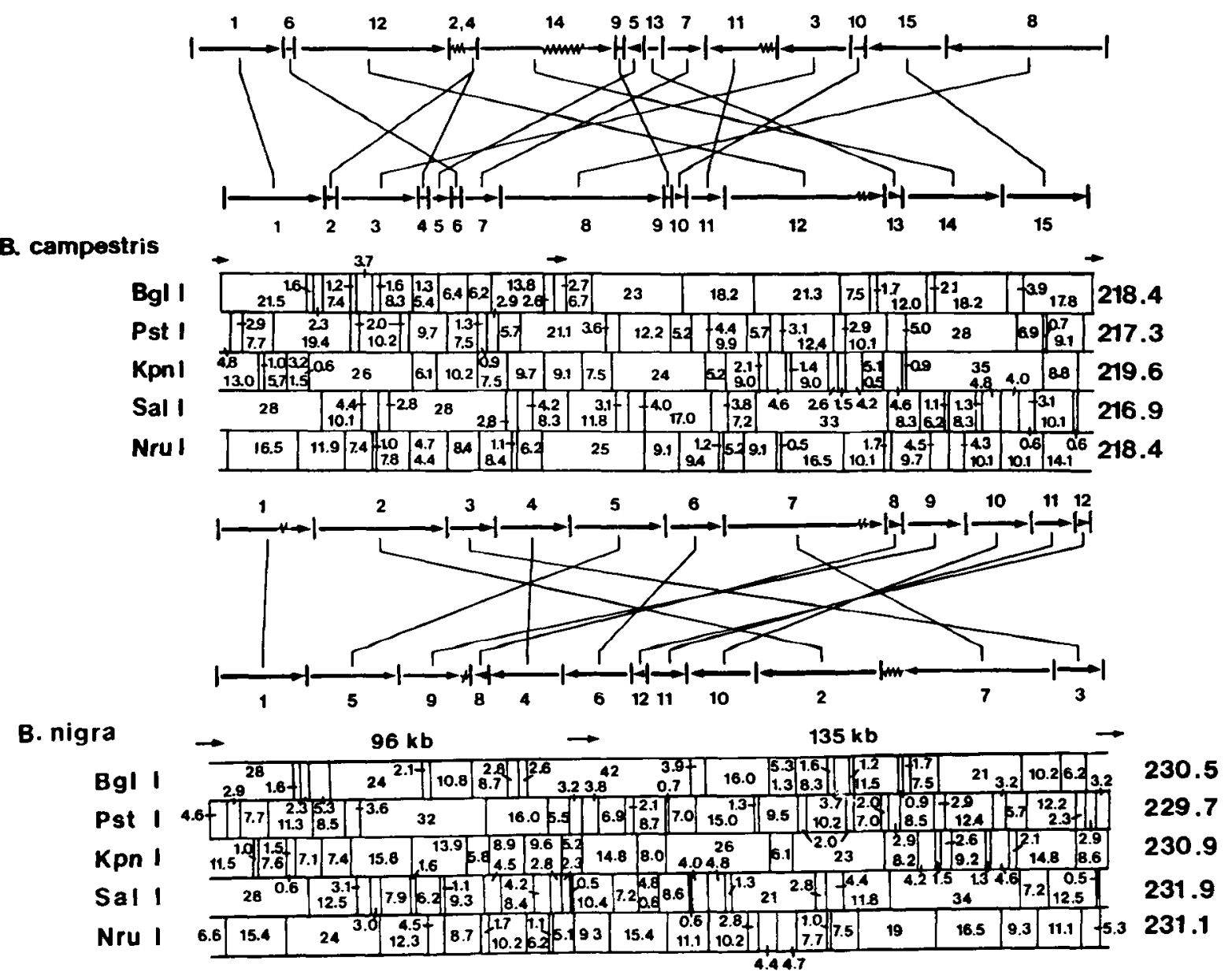

Fig. 5. Relative arrangement of similar sequences in three highly rearranged Brassica mtDNAs. Restriction site maps for $B$. nigra and $R$. sativa (fertile radish) are from Palmer and Herbon (1986); the map for B. campestris is from Palmer and Shields (1984). Arrows directly above the three restriction site maps indicate recombination repeats. Numbered horizontal arrows above and below the $B$. campestris map indicate blocks of sequences whose arrangement has been conserved relative to the genomes of $R$. sativa and $B$. nigra, respectively. The crossing lines connect these cross-hybridizing, arrangement-conserved regions. Squiggles in the arrows indicate sequences found in only one genome in each comparison. Restriction fragment summations are given at the right of the maps.

pared. Mutations were observed at only 2 of these 133 sites (Fig. 1).

To obtain a more accurate measure of divergence, we next compared all six mtDNAs with the 5 enzymes used for mapping and with 15 additional enzymes, AvaI, BamHI, BanI, BanII, BclI, BglII, ClaI, EcoRI, EcoRV, HaelI, HindIII, NciI, NsiI, $\mathrm{XbaI}$, and $\mathrm{XmnI}$. In order to study restriction site divergence without the confounding effects of rearrangement, we focused our attention on unrearranged regions of the genome. Plasmids containing
19 PstI fragments from B. campestris mtDNA, which cover $130 \mathrm{~kb}$ of the genome that is colinear among the three most closely related mtDNAs, were serially hybridized to filter blots containing all six mtDNAs doubly digested with PstI and each of the above enzymes. Double digests were analyzed in order to reduce the size of the fragments being compared and therefore allow better resolution of point mutations from small length mutations. Furthermore, because the PstI sites defining the 19 probe fragments are, with two exceptions, absolutely conserved in all six 
Table 1. Sequence differences among Brassica mtDNAs and cpDNAs

\begin{tabular}{|c|c|c|c|c|c|c|}
\hline & campestris & napus & oleracea & sativa & nigra & hirta \\
\hline campestris & - & $3 / 4002$ & $5 / 4002$ & $5 / 1900$ & $9 / 1759$ & $8 / 2163$ \\
\hline napus & $0.07 / 0.33$ & - & $4 / 4002$ & $5 / 1900$ & $9 / 1759$ & $8 / 2163$ \\
\hline oleracea & $0.12 / 0.30$ & $0.10 / 0.33$ & - & $4 / 1900$ & $8 / 1759$ & $7 / 2163$ \\
\hline sativa & $0.26 / 1.00$ & $0.26 / 1.00$ & $0.21 / 1.00$ & - & $10 / 1496$ & $10 / 1900$ \\
\hline nigra & $0.51 / 2.49$ & $0.51 / 2.49$ & $0.45 / 2.49$ & $0.67 / 2.20$ & - & $14 / 1759$ \\
\hline hirta & $0.37 / 2.08$ & $0.37 / 2.08$ & $0.32 / 2.08$ & $0.53 / 1.90$ & $0.80 / 1.00$ & - \\
\hline
\end{tabular}

The number of restriction site mutations between any two mtDNAs is given in the upper right-hand section of the matrix along with the number of base pairs compared (these numbers take into account the sites cut by both KpnI and BanI). Values in the lower lefthand section correspond to the percentage sequence difference between pairs of mtDNAs (left of slash) and cpDNAs (right of slash) and are calculated as $100 p$, where $p$ is the estimated number of substitutions per base pair from Eq. 3 of Brown et al. (1979). The mtDNA data are from this study and the cpDNA data are from Palmer et al. (1983a)

genomes, we were better able to compare regions lying very close to breakpoints without any of the confounding effects of the rearrangements.

The six Brassica mtDNAs are very similar in nucleotide sequence as estimated by their proportion of shared restriction sites (Table 1). The most divergent pair of species are over $99 \%$ identical in primary sequence, whereas the three most closely related genomes, those of $B$. campestris, $B$. oleracea, and $B$. napus, are roughly $99.9 \%$ identical. Moreover, even these very low sequence divergence values $(0.07-0.80 \%$; Table 1$)$ may be overestimated, because some of the site changes could be due to very small length mutations rather than base substitutions. Therefore, our estimates of sequence divergence should be considered maximal ones.

The entire, roughly $220-\mathrm{kb}$ genomes of three species (B. campestris, B. oleracea, and $B$. napus) were compared with the five enzymes used in mapping, and $130 \mathrm{~kb}$ of each genome was compared with the 15 additional enzymes, producing a total sample size of 675 restriction sites and $4002 \mathrm{nu}-$ cleotide pairs, or almost $2 \%$ of the genome. At the other extreme, only about one-fifth $(45.6 \mathrm{~kb})$ of the $B$. nigra and $R$. sativa mitochondrial genomes could be compared, producing a sample size of 253 sites and $1496 \mathrm{bp}$. Because roughly equal proportions of site mutations were observed in the genomewide comparisons of the three closely related Brassica species as in the more extensive sampling of their unrearranged regions, it appears that rearranged and unrearranged regions are equally susceptible to point mutations. Hence, the limited (in physical terms) sampling of the more rearranged genomes should not produce any bias in detection of point mutations.

\section{Size Variation and Length Mutations}

An accurate assessment of length mutations (deletions/insertions) over the entire mitochondrial ge- nome was possible only for the three most similar mtDNAs, which are also very similar in overall size (218-221 kb; Fig. 1). Length mutations among these three mtDNAs were detected by comparing fragment sizes in two ways - first, by hybridizing clones covering the entire mitochondrial genome to blots containing adjacent lanes of the three mtDNAs digested singly with the five mapping enzymes, and second, by the double digest hybridizations described in the preceding section. This latter assay was more sensitive than the first because the average fragment being compared was smaller. Thus, our study was biased to detect small length mutations in this unrearranged $130-\mathrm{kb}$ portion of the genome.

A minimum of eight length mutations, ranging in size from $50 \mathrm{bp}$ to $6 \mathrm{~kb}$, distinguishes among the mtDNAs of $B$. campestris, B. oleracea, and B. napus. Six of the eight length mutations distinguish the $B$. napus genome from the other two genomes (Fig. 1), and three of these mutations are fairly large $(3,5$, and $6 \mathrm{~kb}$ ), so that the absolute value of these size differences far exceeds the overall genome size difference. For example, the 2-kb size difference between the 221-kb $B$. napus and 219-kb B. oleracea genomes reflects the combined insertion and deletion of over $14 \mathrm{~kb}$ of DNA in a minimum of seven separate events. In contrast, the $B$. campestris and $B$. oleracea genomes differ by only $550 \mathrm{bp}$ in size and by only two detectable length mutations.

The other mitochondrial genomes cover a much wider range of sizes $-208 \mathrm{~kb}$ for $B$. hirta (Palmer and Herbon 1987), and $231 \mathrm{~kb}$ for $B$. nigra and 242 kb for $R$. sativa (Palmer and Herbon 1986). Rearrangement and size variation in these genomes were too extensive to allow analysis of most length mutations. However, Fig. 5 does show the largest length mutations in the $B$. nigra and $R$. sativa genomes relative to that of $B$. campestris. These are of two types, sequence duplications (creating recombination repeats; Palmer and Herbon 1986) and deletions/insertions of unique sequences (Fig. 5). 


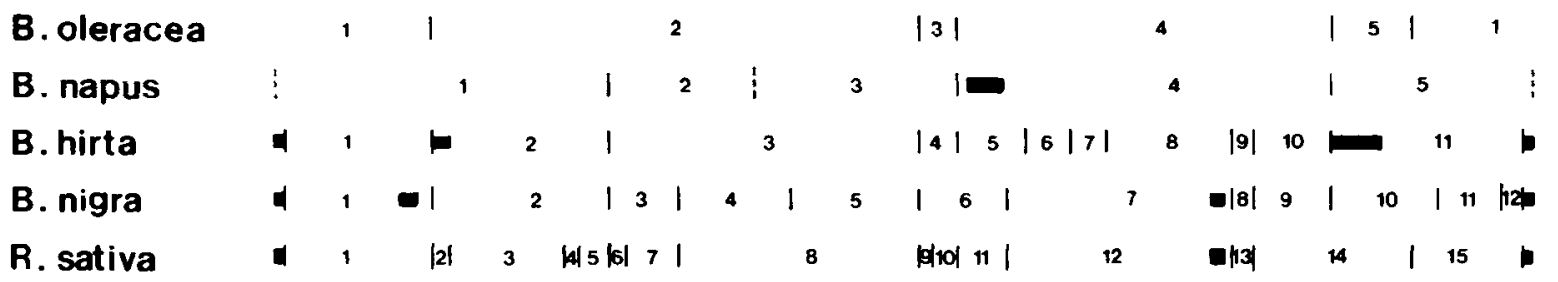

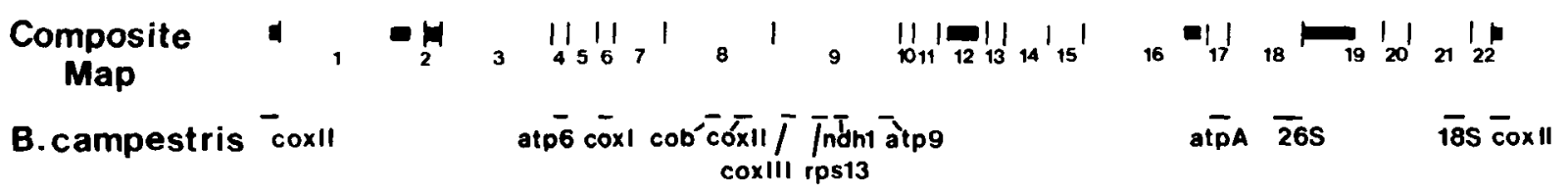

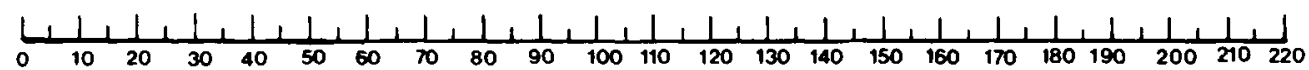

Fig. 6. Summary of mtDNA rearrangement breakpoints relative to the reference genome, from $B$. campestris. Each individual species map shows its rearrangement breakpoints (indicated with horizontal slashes), deletions larger than $1 \mathrm{~kb}$ in size (indicated with solid boxes), and blocks of unrearranged sequences (numbered regions between breakpoints) relative to the map of $B$. campestris (cf. Figs. 3 and 5). The composite map summarizes rearrangements and deletions for all five comparisons. The $B$. campestris gene map is from Makaroff and Palmer (1987). Size scale is in kilobases.

\section{Discussion}

\section{General Features of mtDNA Rearrangement in Brassica}

This study is the first to measure mutation rates over an entire plant mitochondrial genome and documents a unique tempo and mode of evolution for plant mitochondrial DNA. Nucleotide substitutions occur at a very slow rate in these Brassica mtDNAs, whereas internal rearrangements, predominantly inversions, occur frequently. Thus, the Brassica mitochondrial genome may be viewed as a collection of unchanging sequences whose relative arrangement is extremely fluid.

The overall extent and nature of mtDNA rearrangements in Brassica are summarized in a spatial framework in Fig. 6 and in a temporal, phylogenetic framework in Fig. 7. Figure 6 shows that many of the rearrangement breakpoints found in the five genomes that were compared to the $B$. campestris genome map to the same approximate locations relative to $B$. campestris. This probably reflects two factors. First, as discussed in the Results section on inversions, many rearrangements that occurred independently in time are likely to have been fostered by recombination at a limited number of shared endpoint sequences. Second, some rearrangements undoubtedly occurred in the common ancestors of two or more of these species. Unfortunately, the complexity of rearrangement in these genomes, together with the apparent high incidence of shared rearrangement breakpoints, makes impossible an unambiguous determination of phylogenetic direction of individual rearrangement events. It is for this reason that most rearrangements are portrayed with ambiguous polarities in the phylogeny depicted in Fig. 7.

The composite rearrangement map shown in Fig. 6 reveals that rearrangement breakpoints occur on average every $10 \mathrm{~kb}$ in the 208-257-kb Brassica mitochondrial genome and that the longest block of unrearranged sequences (no. 9) is only about $10 \%$ of the length of the genome. Many of the identified plant mitochondrial genes map singly within individual rearrangement units and several of these genes (atp6, coxI, atpA) are closely flanked on both sides by rearrangements in one genome or another. Furthermore, although $c o b$ and $c o x \mathrm{II}$ are linked in all Brassica genomes, they are located far apart in mtDNAs of maize (Lonsdale 1988) and sunflower (Siculella and Palmer 1988). In addition, of the four genes (coxIII, rps 13, ndh 1, atp9) that remain linked on sequence block 9 in the composite Brassica map (Fig. 6), only two, rps 13 and $n d h 1$, are also linked in such plants as maize, tobacco, and sunflower (Bland et al. 1986; Lonsdale 1988; Siculella and Palmer 1988). In general, then, gene order is inconsequential to the proper function of plant mitochondrial genes.

There are two indications that rearrangement has been most extensive in the lineage leading to radish, $R$. sativa. First, the fertile radish line examined in this study is more rearranged relative to $B$. campestris than any other Brassica species, including two species ( $B$. nigra and $B$. hirta) that are phylogenetically more distantly related (Fig. 7). Second, the mtDNAs of this fertile radish line and a cytoplasmic male sterile (CMS) radish line have been shown to be extensively rearranged (approx. 10 inversions), in spite of a high degree of primary se- 


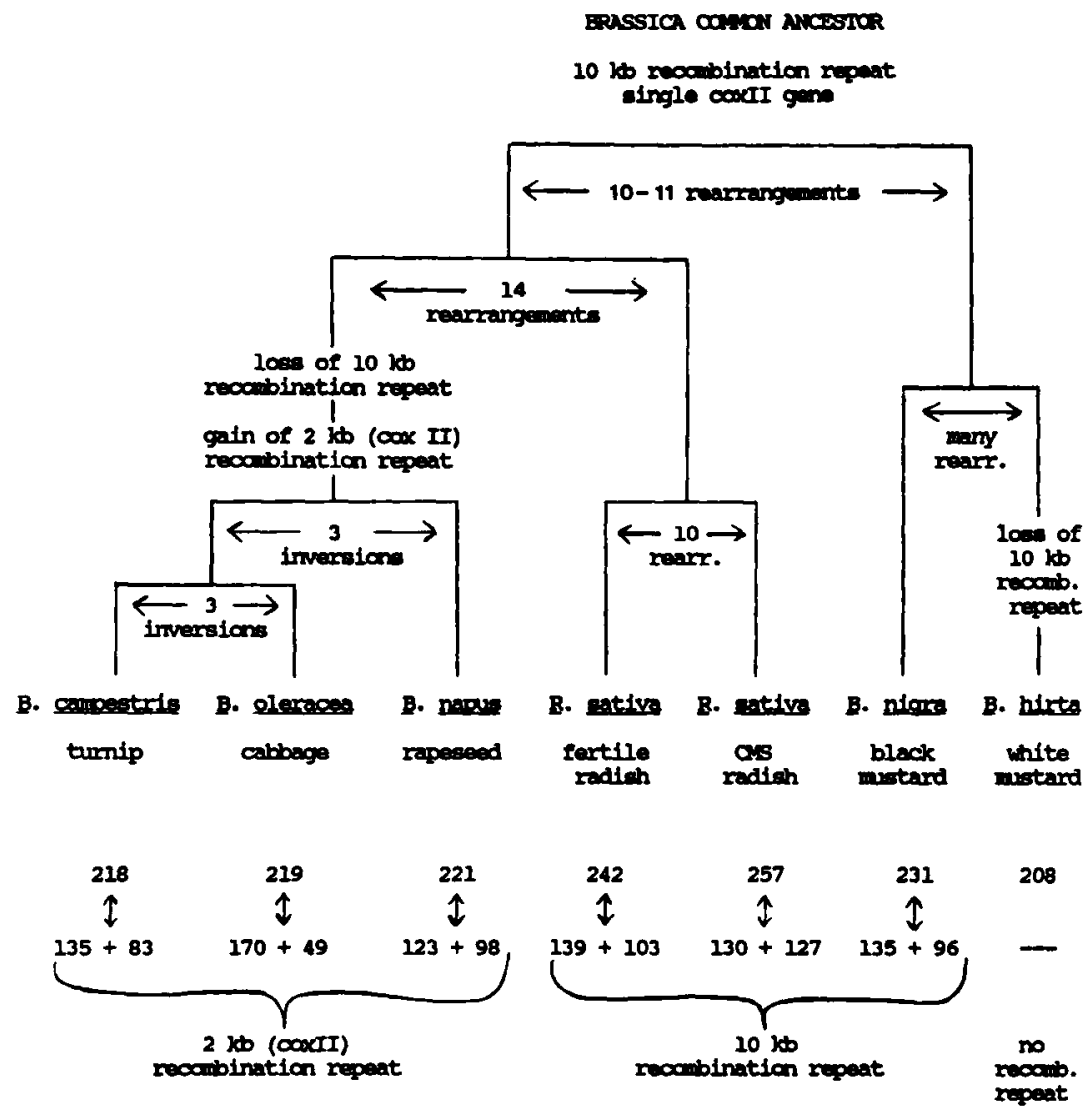

Fig. 7. Phylogenetic history of mtDNA rearrangements in Brassica. Top Cytoplasmic phylogeny for Brassica based on cpDNA restriction site mutations (Palmer et al. 1983a). This phylogeny is cladistically derived and is not intended to convey divergence times. Numbers of rearrangments are given relative to the reference genome, $B$. campestris, except for the 10 rearrangements that have been shown (Makaroff and Palmer 1988) to distinguish the CMS and fertile mtDNAs of $R$. sativa. Bottom Sizes of master chromosomes and (where present) subgenomic circles resulting from high frequency recombination at the indicated recombination repeats. quence similarity in both mtDNA and cpDNA of the two radish lines (Makaroff and Palmer 1988). We are presently sequencing the endpoints of a number of these radish-derived rearrangements in order to examine mutations potentially involved in CMS; these studies may also provide clues concerning the greater instability of mtDNA in radish.

Although it is impossible to polarize Brassica mtDNA inversions, the evolutionary direction of several large insertions and deletions can be inferred with reasonable confidence. In particular, the duplication of the coxII gene as a recombination repeat is a relatively recent event in Brassica evolution, occurring in the common ancestor of the three most closely related species examined, B. campestris, $B$. oleracea, and $B$. napus (Fig. 7). The duplication represented by the $10-\mathrm{kb}$ recombination repeat is present in only some taxa in each of the two major clades of the Brassica phylogeny (Fig. 7). We favor the idea, expressed in Fig. 7, that this duplication was present in the common ancestor of the genus and was subsequently lost twice independently - once in $B$. hirta and once in the 2-kb recombination repeat-containing lineage. We prefer this explanation to the equally parsimonious one of two parallel gains, once in $B$. nigra and once in $R$. sativa. It seems easier to imagine parallel loss of one copy of a duplicate (and hence nonessential) sequence than its parallel duplication in a genome of the size and complexity of the Bras- sica mitochondrial genome. Although these large duplications in plant mtDNA are relatively ephemeral compared to those in cpDNA, which can persist for several hundred million years (Palmer 1985a,b), they are long-lived relative to tandem duplications in animal mtDNA, which are usually restricted to particular individuals or populations (Moritz and Brown 1987).

\section{Novel Mode of Evolution of Plant mtDNA}

Comparisons of plant mtDNA with cpDNA and animal mtDNA reveal striking contrasts in genome evolution. The much smaller mtDNAs of animals are extremely invariant in genome arrangement (all vertebrate mtDNAs have the same gene order) yet undergo extraordinarily rapid changes in primary sequence (Brown 1983, 1985; Wilson et al. 1985). The mean intraspecific divergence in animal mtDNA ranges from $0.18 \%$ to $4.10 \%$ within 12 different mammalian species (Wilson et al. 1985). This compares to a mean interspecific divergence of $0.37 \%$ (Table 1) and an intraspecific divergence of $0 \%$ (Palmer 1988) in Brassica mtDNAs. We estimate plant mtDNA sequence change to be on the order of 100 times slower than for animal mtDNA. This is based on calculations that cpDNA mutates 10100 times slower than animal mtDNA (Zurawski et al. 1984) and on our own findings that mtDNA in 
Brassica mutates 4 times slower than cpDNA (see below). Overall then, plant and animal mtDNA evolution are completely opposite with respect to both primary sequence and genome arrangement.

Gene order is almost as highly conserved in land plant cpDNA as in animal mtDNA (Palmer 1985a,b; Gray 1986) and is therefore orders of magnitude more constrained than in plant mtDNA. Indeed, we earlier demonstrated (Palmer et al. 1983a) complete colinearity among the cpDNAs of the six Brassica species whose mtDNAs are shown to be so highly rearranged (Figs. 3 and 5). This disparity in rearrangement rates for the two plant cytoplasmic genomes far exceeds their difference in point mutation rates. Comparisons among the same Brassica species reveal that substitution frequencies across the genome are about four times slower for plant mtDNA than for cpDNA (Table 1). This number, which is based on indirect sampling of substitutions by restriction site analysis, is in excellent agreement with the recent estimate of a threefold slower rate of synonymous substitutions for plant mitochondrial genes compared to chloroplast genes (Wolfe et al. 1987).

Our total genome divergence estimates and those based on single genes (Wolfe et al. 1987) are in disagreement with those of McClean and Hanson (1986). They estimated nucleotide substitutions based on the shared fragment method, which assumes that all fragment changes are due to substitutions. However, they pointed out that their estimates would not reflect actual accumulation of substitutions if the mitochondrial genomes contained many rather than a few rearrangements. The assumption of few rearrangements is clearly not met for Brassica mtDNAs, nor apparently for those in Zea (Sederoff et al. 1981; Schardl et al. 1985; Dewey et al. 1986) and in Nicotiana (Bland et al. 1985) and Petunia (Young and Hanson 1987), two genera in the same family as Lycopersicon. Because rearrangement appears to be the major mode of plant mtDNA change, we believe that the Lycopersicon divergence values calculated by McClean and Hanson (1986) are significantly overestimated.

\section{Mechanisms of Plant mtDNA Evolution}

Why does plant mtDNA rearrange so rapidly, yet remain so invariant in primary sequence? The large size of plant mitochondrial genomes and their abundance of noncoding sequences suggest that a high proportion of possible rearrangements is likely to be tolerated, i.e., will not disrupt gene function. Our transcriptional studies indicate that at most only about one-third of the $218-\mathrm{kb}$ mitochondrial genome of $B$. campestris is likely to have coding functions and that almost all genes are transcribed singly (Makaroff and Palmer 1987). It seems reasonable therefore to expect that only $10 \%$ or less of the DNA is genic in the larger plant genomes (Ward et al. 1981). In contrast, land plant cpDNAs are densely packed with genes, many of which are cotranscribed (Gray 1986), whereas animal mtDNA lacks spacer sequences almost entirely and in mammals at least is transcribed into a single genome-sized RNA (Clayton 1984; Brown 1985). Thus, gene packing constraints on rearrangement should be extremely relaxed in plant mtDNA compared to both cpDNA and animal mtDNA. Furthermore, plant mitochondrial genomes seem able to tolerate at least certain rearrangements that actually do affect gene structure; these generate novel, recombinant genes that appear to be involved in cytoplasmic male sterility (Bailey-Serres et al. 1986; Dewey et al. 1986; Young and Hanson 1987).

A second major factor promoting rearrangement in plant mtDNAs is the prevalence of short dispersed repeats that could serve as the points of crossover for homologous recombination events. Such repeats are virtually absent from animal mtDNA and from most cpDNAs (Palmer 1985a,b; Brown 1985). In contrast, several families of short dispersed repeats exist in Brassica mtDNAs (Palmer and Shields 1984; Shirzadegan and Palmer, unpublished data). Furthermore, hybridization studies reveal that these repeats are located near or at the precise breakpoints of the three inversions (Fig. 3) that distinguish mtDNAs of $B$. campestris and $B$. oleracea (M. Shirzadegan and J. Palmer, unpublished data). Sequencing studies are now in progress to clarify the relationship between repeats and inversions in Brassica mtDNA.

The slow rate of point mutations in plant mtDNA is intriguing. The fact that the genomes are mostly noncoding might cause one to expect high levels of sequence variation, particularly when measured by restriction site studies of the entire genome. Yet, plant mtDNA accumulates substitutions $3-4$ and 100 times more slowly than the densely gene-packed DNAs of chloroplasts and animal mitochondria, respectively. We therefore favor the idea that the mutation rate in plant mtDNA is driven by factors affecting mutation pressures, rather than by stringent selection pressures. For example, we predict that plant mitochondria are likely to contain highly efficient systems for repairing damage to their DNA, and perhaps, a relatively error-free DNA replication system. In contrast, the high mutation rate in animal mtDNA has been attributed, at least in part, to faulty replication enzymes and a general absence of DNA repair processes (Brown 1983).

The explanations we have offered for the high rate of rearrangements and low rate of substitutions in plant mtDNA are but prima facie biochemical ones and do not address underlying selective factors. What at present is completely mystifying is why plant mtDNAs can tolerate lots of extra sequences, 
including recombinogenic repeats, whereas animal mtDNAs cannot. Similarly, why can animal mtDNAs tolerate much higher substitution rates than plant mtDNAs, when both encode similar sets of genes?

Acknowledgments. We thank J. Nugent for assistance with the mtDNA isolations, $C$. Shields for assistance with the initial filter hybridizations, and W. Brown, T. Bruns, P. Calie, C. Makaroff, C. Moritz, and M. Zolan for critical reading of the manuscript. This research was funded by NIH grant GM-35087 to J.D. Palmer.

\section{References}

Bailey-Serres J, Hanson DK, Fox TD, Leaver CJ (1986) Mitochondrial genome rearrangement leads to extension and relocation of the cytochrome c oxidase subunit I gene in Sorghum. Cell 47:567-576

Bland MM, Matzinger DF, Levings CS III (1985) Comparison of the mitochondrial genome of Nicotiana tabacum with its progenitor species. Theor Appl Genet 69:535-541

Bland MM, Levings CS III, Matzinger DF (1986) The tobacco mitochondrial ATPase subunit 9 gene is closely linked to an open reading frame for a ribosomal protein. Mol Gen Genet 204:8-16

Broach JR (1982) The yeast plasmid 2 micron circle. Cell 28: 203-204

Brown WM (1983) Evolution of animal mitochondrial DNA. In: Nei M, Koehn RK (eds) Evolution of genes and proteins. Sinauer, Sunderland MA, pp 62-88

Brown WM (1985) The mitochondrial genome of animals. In: MacIntyre RJ (ed) Monographs in evolutionary biology: molecular evolutionary genetics. Plenum, New York, pp 95-130

Brown WM, George M Jr, Wilson AC (1979) Rapid evolution of mitochondrial DNA. Proc Natl Acad Sci USA 76:19671971

Brown WM, Prager EM, Wang A, Wilson AC (1982) Mitochondrial DNA sequences of primates: tempo and mode of evolution. J Mol Evol 18:225-239

Chetrit P, Mathieu C, Muller JP, Vedel F (1984) Physical and gene mapping of cauliflower (Brassica oleracea) mitochondrial DNA. Curr Genet 8:413-421

Clayton DA (1984) Transcription of the animal mitochondrial genome. Annu Rev Biochem 53:573-594

Dewey RE, Levings CS III, Timothy DH (1986) Novel recombinations in the maize mitochondrial genome produce a unique transcriptional unit in the Texas male-sterile cytoplasm. Cell 44:439-449

Gray J (1986) Wonders of chloroplast DNA. Nature 322:501502

Kolodner R, Tewari KK (1972) Physicochemical characterization of mitochondrial DNA from pea leaves. Proc Natl Acad Sci USA 69:1830-1834

Lebacq P, Vedel F (1981) Sal I restriction enzyme analysis of chloroplast and mitochondrial DNAs in the genus Brassica. Plant Sci Lett 23:1-9

Lonsdale DM (1988) The plant mitochondrial genome. In: Davies DD (ed) The biochemistry of plants, vol. II, biochemistry of metabolism. Academic Press, Orlando FL (in press)

Makaroff CA, Palmer JD (1987) Extensive mitochondrial-specific transcription of the Brassica campestris mitochondrial genome. Nucleic Acids Res 15:5141-5156

Makaroff CA, Palmer JD (1988) Mitochondrial DNA rearrangements and transcriptional alterations in the male-sterile cytoplasm of Ogura radish. Mol Cell Biol 8:1474-1480
McClean PE, Hanson MR (1986) Mitochondrial DNA sequence divergence among Lycopersicon and related Solanum species. Genetics 112:649-667

Moritz C, Brown WM (1987) Tandem duplications in animal mitochondrial DNAs: variation in incidence and gene content among lizards. Proc Natl Acad Sci USA 84:7183-7187

Palmer JD (1985a) Evolution of chloroplast and mitochondrial DNA in plants and algae. In: MacIntyre RJ (ed) Monographs in evolutionary biology: molecular evolutionary genetics. Plenum, New York, pp 131-240

Palmer JD (1985b) Comparative organization of chloroplast genomes. Annu Rev Genet 19:325-354

Palmer JD (1986) Isolation and structural analysis of chloroplast DNA. Methods Enzymol 118:167-186

Palmer JD (1988) Intraspecific variation and multicircularity in Brassica mitochondrial DNAs. Genetics 118:341-351.

Palmer JD, Herbon LA (1986) Tripartite mitochondrial genomes of Brassica and Raphanus: reversal of repeat configurations by inversion. Nucleic Acids Res 14:9755-9765

Palmer JD, Herbon LA (1987) Unicircular structure of the Brassica hirta mitochondrial genome. Curr Genet 11:565570

Palmer JD, Shields CR (1984) Tripartite structure of the Brassica campestris mitochondrial genome. Nature 307:437-440

Palmer JD, Shields CR, Cohen DB, Orton TJ (1983a) Chloroplast DNA evolution and the origin of amphidiploid Brassica species. Theor Appl Genet 65:181-189

Palmer JD, Shields CR, Cohen DB, Orton TJ (1983b) An unusual mitochondrial DNA plasmid in the genus Brassica. Nature 301:725-728

Pring DR, Lonsdale DM (1985) Molecular biology of higher plant mitochondrial DNA. Int Rev Cytol 97:1-46

Schardl CL, Pring DR, Lonsdale DM (1985) Mitochondrial DNA rearrangements associated with fertile revertants of S-type male-sterile maize. Cell 43:361-368

Sederoff RR (1987) Molecular mechanisms of mitochondrialgenome evolution in higher plants. Am Nat 130:S30-S45

Sederoff RR, Levings CS III, Timothy DH, Hu WWL (1981) Evolution of DNA sequence organization in mitochondrial genomes of Zea. Proc Natl Acad Sci USA 78:5953-5957

Siculella L, Palmer JC (1988) Physical and gene organization of mitochondrial DNA in fertile and male sterile sunflower: CMS-associated alterations in structure and transcription of the atpA gene. Nucleic Acids Res 16:3787-3799

Stern DB, Palmer JD (1984) Recombination sequences in plant mitochondrial genomes: diversity and homologies to known mitochondrial genes. Nucleic Acids Res 12:6141-6157

Ward BL, Anderson RS, Bendich AJ (1981) The mitochondrial genome is large and variable in a family of plants (Cucurbitaceae). Cell 25:793-803

Wilson AC, Cann RL, Carr SM, George M, Gyllensten UB, HelmBychowski KM, Higuchi RG, Palumbi SR, Prager EM, Sage RD, Stoneking M (1985) Mitochondrial DNA and two perspectives on evolutionary genetics. Biol J Linn Soc 26:375400

Wolfe KH, Li W-H, Sharp PM (1987) Rates of nucleotide substitution vary greatly among plant mitochondrial, chloroplast, and nuclear DNAs. Proc Natl Acad Sci USA 84:9054-9058

Young EG, Hanson MR (1987) A fused mitochondrial gene associated with cytoplasmic male sterility is developmentally regulated. Cell 50:41-49

Zurawski G, Clegg MT (1987) Evolution of higher-plant chloroplast DNA-encoded genes: implications for structure-function and phylogenetic studies. Annu Rev Plant Physiol 38: $391-418$

Zurawski G, Clegg MT, Brown AHD (1984) The nature of nucleotide sequence divergence between barley and maize chloroplast DNA. Genetics 106:735-749

Received March 2, 1988/Revised and accepted April 7, 1988 